\title{
Analytical study of the thermal behaviour and stability of a small satellite
}

\author{
Isabel Pérez-Grande / Angel Sanz-Andrés / Carmen Guerra / Gustavo Alonso
}

A B S T R A C T

\begin{abstract}
In this work the thermal analysis of a small satellite orbiting around the Earth has been approached by direct integration of the heat balance equations of a two-node reduced model, obtaining a linearized sec-ond order ODE problem, similar in form to the classical case of the forced vibration of a damped system. As the thermal loads (solar radiation, albedo, etc.) are harmonic, the problem is solved by means of Fou-rier analysis methods. Research on that field can be directly applied to the analysis of thermal problems and the results obtained are satisfactory. Working on the frequency domain streamlines the analysis, simplifies the study and facilitates the experimental testing. The transfer functions are obtained for the two-node case but the study can be extended to an $n$-node model.
\end{abstract}

\section{Introduction}

The thermal control plays an increasingly relevant role in the design process of spacecraft. Over the last few decades, a big effort has been made in the field of spacecraft thermal control to develop both new technologies and a number of software tools used to facilitate the complex tasks of thermal calculations. The first step in the calculation of temperatures is to determine the thermal loads onto the system and the conductive and radiative couplings. A good compendium of technologies, thermal modelling and thermal data can be found in [1]. However, the widespread use of these software tools based on numerical methods has, as a consequence, that nowadays analytical methods for thermal analysis of satellites are seldom used. Nevertheless, analytical studies highlight the physics of the problem and are essential at an early stage of the thermal design when the mission and structure are not yet completely defined. At the beginning of spacecraft thermal control development, some attempts were made to face the problem of thermal design of a spacecraft analytically. For instance, Oshima and Oshima [2] tried to deal with the problem but the results were not easy to apply mainly due to the parameters used and the complexity of the necessary calculations. More recently, Arduini et al. [3] presented a new approach for solving the inverse problem by means of linearized techniques and Gadalla [4] studied analytically the temperature in a rotating cylindrical spacecraft.

The aim of this paper is to present a simple analytical method to study the temperature variations and thermal stability of small (micro and nano) compact spinning satellites with the solar panels mounted onto the body of the spacecraft. Over the last decade, the number of launches of this type of small satellites has notably increased, highlighting the importance of the availability of analytical tools that can help in the design process. Since these satellites have outer temperatures quite uniform due to the homogenisation effect of the spinning, and the inner equipment has different thermal requirements than the shell, they can be thermally modelled by means of just two nodes, the inner one, which includes all the equipment within it (payload and electronics), and the outer one, representing the shell, the solar panels and any external device located on the outer surface of the satellite. In the following sections, the temperature of both nodes is studied throughout the orbit solving a second order ordinary differential equation. The thermal stability, that is the thermal response to random fluctuations of the thermal loads, is studied through Fourier analysis, technique frequently used in vibration studies [5] but seldom applied to thermal verification. Barbagallo and Stramaccioni [6] describe this method and its suitability when only the thermal stability of spacecraft within a certain bandwidth is of interest. As an example, they refer to a thermal requirement of the LISA mission, which imposes a limitation on the temperature fluctuations on the optical bench: they have to be kept below $10^{-6} \mathrm{~K} / \sqrt{\mathrm{Hz}} @ 10^{-3} \mathrm{~Hz}$.

\section{Thermal model}

As has been stated in the previous section, a small satellite can be thermally modelled with just two nodes. Let $C_{i}$ and $C_{s}$ be the thermal capacities of the inner and the outer nodes, respectively, and $T_{i}$ and $T_{s}$ their temperatures. The geometric model corresponding to this thermal mathematical model is shown in Fig. 1. Both nodes are thermally coupled, both by conduction and radiation. Let $k_{i s}$ be the conductive coupling coefficient and $r_{i s}$ the radiative coupling coefficient, defined in such a way that the energy balance equations for both nodes are 


\section{Nomenclature}

$\begin{array}{ll}a & \text { albedo coefficient } \\ A & \text { area } \\ C & \left.\text { heat capacity [J K }{ }^{-1}\right] \\ E & \text { damping parameter } \\ G_{s} & \left.\text { solar constant [W m }{ }^{-2}\right] \\ H & \text { ratio of thermal couplings } \\ h_{i s}, h_{s s} & \text { thermal coupling coefficients }\left[\mathrm{W} \mathrm{K}^{-1}\right] \\ k_{i s} & \text { conductive coupling coefficient }\left[\mathrm{W} \mathrm{K}^{-1}\right] \\ P & \text { period [s] } \\ \dot{Q} & \left.\text { heat flux [W } \mathrm{W}^{-2}\right] \\ r_{i s} & \text { radiative coupling coefficient }\left[\mathrm{W} \mathrm{K}^{-4}\right]\end{array}$

$\begin{array}{ll}t & \text { time [s] } \\ T & \text { temperature }[\mathrm{K}] \\ \alpha_{\mathrm{s}} & \text { solar absorptivity } \\ \varepsilon & \text { emissivity } \\ \varphi & \text { phase delay } \\ \theta & \text { dimensionless temperature } \\ \Theta & \text { fourier transform of the dimensionless temperature } \\ \sigma & \text { stefan-Boltzmann constant }\left[\mathrm{W} \mathrm{m}^{-2} \mathrm{~K}^{-4}\right] \\ \tau & \text { dimensionless time } \\ \omega & \text { dimensionless frequency } \\ \zeta & \text { damping factor }\end{array}$

$$
\begin{aligned}
& C_{s} \dot{T}_{s}=\dot{Q}_{s o l}+\dot{Q}_{a l b}+\dot{Q}_{p}+k_{i s}\left(T_{i}-T_{s}\right)+r_{i s}\left(T_{i}^{4}-T_{s}^{4}\right)-A_{s c} \varepsilon \sigma T_{s}^{4} \\
& C_{i} \dot{T}_{i}=\dot{Q}_{i}-k_{i s}\left(T_{i}-T_{s}\right)-r_{i s}\left(T_{i}^{4}-T_{s}^{4}\right)
\end{aligned}
$$

where $A_{S C}$ is the spacecraft's outer shell area; $\varepsilon$ is its emissivity; $\sigma=5.67 \times 10^{-8} \mathrm{~W} \mathrm{~m}^{-2} \mathrm{~K}^{-4}$ is the Stefan-Boltzmann constant; $\dot{Q}_{s o l}$ and $\dot{Q}_{a l b}$ represent the solar irradiation and the planetary albedo thermal loads on the outer surface node, respectively; $\dot{Q}_{p}$ is the planetary infrared radiation, and $\dot{Q}_{i}$ represents the heat dissipation in the inner node.

Regarding $\dot{Q}_{s o l}$, it will be zero during the fraction of orbit in eclipse, which depends on the $\beta$-angle (minimum angle between the orbital plane and the solar vector) as described in Ref. [1]. During the fraction of orbit while the satellite is illuminated, the solar heat on it will be $\dot{Q}_{s o l, i l}=G_{s} A_{s c-p} \alpha_{s}$, being $G_{s}$ the mean solar irradiation, for an Earth orbit $G_{s}=1370 \mathrm{~W} \mathrm{~m}^{-2}, \alpha_{s}$ the solar absorptivity of the outer shell of the satellite, and $A_{s c-p}$ the spacecraft surface projected in the Sun's direction. Since this area remains practically constant for small compact satellites (spherical or poliedrical shape) then the solar heat load will be constant during this period. The solar heat load on the satellite can therefore be written as

$\dot{\mathrm{Q}}_{\mathrm{sol}}=\mathrm{G}_{\mathrm{S}} A_{\mathrm{sc}-p} \alpha_{\mathrm{s}} f_{1}(\boldsymbol{t})=\dot{\mathrm{Q}}_{\mathrm{s} 1} f_{1}(\boldsymbol{t})$

where $f_{1}(t)$ represents the day-to-night variations of the solar irradiation and, as described above, has a square wave shape. This function is shown in Fig. 2, where $P_{i l}$ and $P_{o r b}$ are the illumination and the orbital period, respectively.

Concerning the albedo thermal loads on the outer node, in general they depend on the orbit altitude and the solar zenith angle at satellite position. For LEO orbits, albedo loads can be estimated using a function that only depends on the angle between the Sun

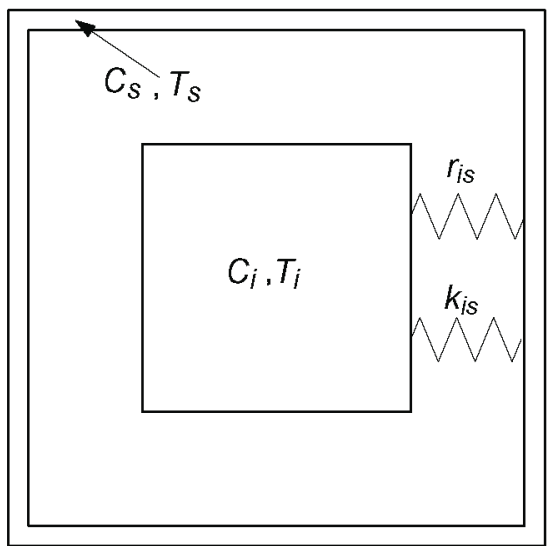

Fig. 1. Sketch of the geometric model corresponding to the two-node mathematical model. vector and the satellite position vector. Thus, the albedo heat input can be written as

$\dot{Q}_{a l b} \cong a G_{s} A_{s c} F_{s c-p} \alpha_{s} f_{2}(t)=\dot{Q}_{s 2} f_{2}(t)$

where $A_{s c}$ is the area of the whole satellite and $F_{S c-p}$ is the view factor from the whole satellite to the planet in order to make Eq. (2) consistent. The term $f_{2}(t)$ represents the day-to-night variations of the albedo thermal loads, maximum when the solar vector coincides with the local vertical, and practically zero when the angle between these two vectors is $90^{\circ}$. It is also zero during the eclipse period. Therefore it can be mathematically written as $f_{2}(t)=$ $\cos (2 \pi t / P)$ for $-\frac{\pi}{2} \leqslant \alpha \leqslant \frac{\pi}{2}$ and $f_{2}(t)=0$ for the rest of the orbit. This method of evaluating the albedo thermal loads can be found in [7]. The shape of the function $f_{2}(t)$ is also shown in Fig. 2 .

The planetary infrared radiation can be calculated as $\dot{Q}_{p}=\varepsilon A_{s c} F_{s c-p} \sigma T_{p}^{4}, T_{p}$ being the planet blackbody-equivalent temperature.

The integration of Eq. (1) has been carried out using a fourth-order Runge Kutta algorithm to obtain the temperatures of both nodes as a function of time. A large enough number of orbits have been calculated in order to obtain the periodic solution. The temperature of the outer node as a function of the temperature of the inner node is shown in Fig. 3 for the values of the parameters given in Table 1 . The period, $5800 \mathrm{~s}$, corresponds to an orbit altitude of $600 \mathrm{~km}$. The values of $\alpha_{s}$ and $\varepsilon$ have been chosen considering that $85 \%$ of the satellite is covered with solar cells $\left(\alpha_{s}=0.75, \varepsilon\right.$ $=0.82)$ and $15 \%$ with white paint $\left(\alpha_{s}=0.23\right.$ and $\left.\varepsilon=0.88\right)$. The cycle is anticlockwise and note that when $T_{i}>T_{s}$ (points above the line

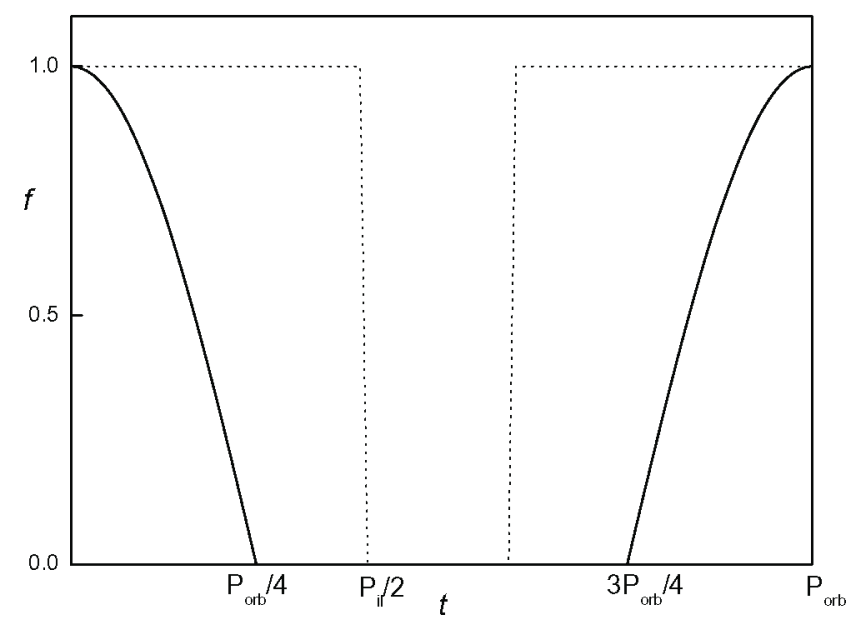

Fig. 2. Typical variation with time of the solar and planetary albedo thermal loads on the skin of a satellite orbiting around a planet. Dotted line: solar irradiation. Solid line: planetary albedo radiation. 


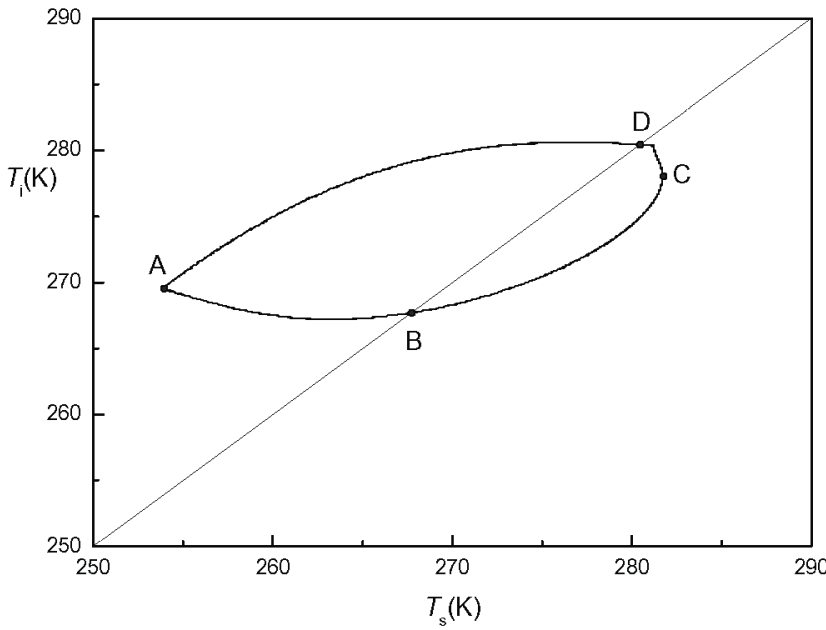

Fig. 3. Inner node's temperature $T_{i}$ versus outer node's temperature $T_{s}$ for the parameters' values given in Table 1.

Table 1

Parameter values used to calculate the temperature variations along the orbit.

\begin{tabular}{ll}
\hline Outer node area $A_{s}\left(\mathrm{~m}^{2}\right)$ & 3.14 \\
Inner node area $A_{i}\left(\mathrm{~m}^{2}\right)$ & 1.13 \\
Outer node heat capacity $C_{s}\left(\mathrm{~J} \mathrm{~K}^{-1}\right)$ & 30,000 \\
Inner node heat capacity $C_{i}\left(\mathrm{~J} \mathrm{~K}^{-1}\right)$ & 20,000 \\
Conductive coupling coefficient $k_{i s}\left(\mathrm{~W} \mathrm{~K}^{-1}\right)$ & 10 \\
Radiative coupling coefficient $r_{i s}\left(\mathrm{~W} \mathrm{~K}^{-4}\right)$ & $3.6 \cdot 10^{-8}$ \\
Solar constant $G_{s}\left(\mathrm{~W} \mathrm{~m} \mathrm{~m}^{-2}\right)$ & 1360 \\
Earth albedo coefficient $a$ & 0.3 \\
Solar absorptivity $\alpha_{s}$ & 0.67 \\
Emissivity $\varepsilon$ & 0.83 \\
Earth blackbody-equivalent temperature $(\mathrm{K})$ & 259 \\
Orbital period $P_{\text {orb }}(\mathrm{s})$ & 5800 \\
Ratio $P_{i l} / P_{o r b}$ & 0.63 \\
Internal heat dissipation $\dot{Q}_{i}(\mathrm{~W})$ & 50 \\
\hline
\end{tabular}

$T_{i}=T_{s}$, indicated in the figure) the net heat flux is towards the satellite shell and viceversa. This curve can be better explained if the variation of both temperatures $T_{i}$ and $T_{s}$ is plotted as a function of time, as shown in Fig. 4, where the characteristic points of the cycle followed by the outer node are outlined as explained below (A: sunrise; C: sunset; B,D $T_{i}=T_{s}$ ). Since the internal dissipation

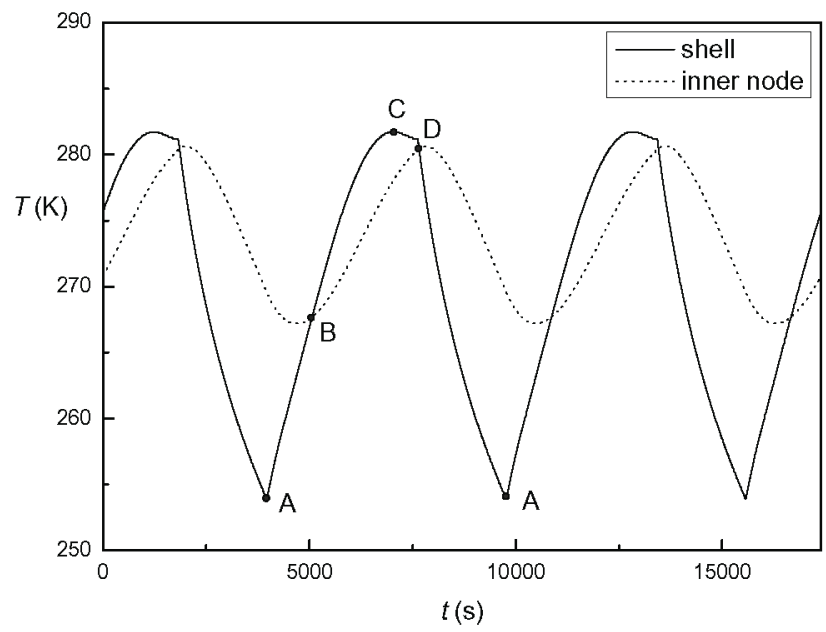

Fig. 4. Inner and outer nodes' temperatures as a function of time for the parameters' values given in Table 1. at the inner node is assumed to be constant, the variation of temperature of this node is due to the thermal coupling with the outer node, which is affected by the highly variable external thermal loads. The temperatures of both nodes coincide in the points indicated as $B$ and $D$. When the satellite enters the eclipse region of the orbit (C), the temperature of the outer node (C-D-A) decreases faster than the temperature of the inner node. A delay in the response of the inner node due to the finite thermal coupling between nodes can also be seen in Fig. 4. The results shown in Fig. 4 can be compared with those obtained in Ref. [8], where it can be observed that the shape of the day-to-night variations of temperature have the same profile as those obtained in this paper.

The influence of the parameters of Eq. (4) could be found by means of a parametric analysis. Unfortunately, due to the great number of parameters involved in the problem it is not easy to obtain general conclusions from such analysis. Nevertheless, some interesting results can be obtained if some simplifications are introduced and the equations are appropriately rearranged.

The first step to simplify the equations is to linearize the radiative coupling term. Let $T_{m}=\left(T_{i}+T_{s}\right) / 2$ be the mean value of both nodes' temperatures. The radiative term can be rewritten as $r_{i s}\left(T_{i}^{4}-T_{s}^{4}\right) \simeq r_{i s} 4 T_{m}^{3}\left(T_{i}-T_{s}\right)$ provided that both $\left(\left(T_{s}-T_{m}\right) / T_{m}\right)^{2} \ll 1$ and $\left(\left(T_{m}-T_{i}\right) / T_{m}\right)^{2}<<1$.

Then, Eqs. (1a) and (1b) can be rewritten as

$$
\begin{aligned}
& C_{s} \dot{T}_{s}=\dot{Q}_{s 1} f_{1}(t)+\dot{Q}_{s 2} f_{2}(t)+\dot{Q}_{p}+h_{i s}\left(T_{i}-T_{s}\right)-A_{s c} \varepsilon \sigma T_{s}^{4} \\
& C_{i} \dot{T}_{i}=\dot{Q}_{i}-h_{i s}\left(T_{i}-T_{s}\right)
\end{aligned}
$$

where $h_{\text {is }}=k_{\text {is }}+4 r_{i s} T_{m}^{3}$ is the linearized thermal coupling between nodes.

The next step will be to expand $T_{i}(t)$ and $T_{s}(t)$ around the mean orbital temperatures $\bar{T}_{s}$ and $\bar{T}_{i}$.

\section{Mean equilibrium temperatures}

The mean equilibrium temperatures are obtained assuming that the satellite orbit remains constant. With this hypothesis $T_{S}$ and $T_{i}$ will be periodic in time, with the same period as the orbit, $P_{o r b}$, so that,

$T_{s}(t)=T_{s}\left(t+P_{\text {orb }}\right)=\bar{T}_{s}+\Delta T_{s}$

$T_{i}(t)=T_{i}\left(t+P_{o r b}\right)=\bar{T}_{i}+\Delta T_{i}$

where $\Delta T_{s}$ and $\Delta T_{i}$ are the temperature fluctuations around the average orbital temperatures $\bar{T}_{S}$ and $\bar{T}_{i}$, which are given by

$\bar{T}_{s}=\frac{1}{P_{\text {orb }}} \int_{t}^{t+P_{\text {orb }}} T_{s} \mathrm{~d} t$
$\bar{T}_{i}=\frac{1}{P_{\text {orb }}} \int_{t}^{t+P_{\text {orb }}} T_{i} \mathrm{~d} t$

Integrating Eqs. (4a) and (4b) over one period and neglecting second order terms $\left(\Delta T_{s} / T_{s}\right)^{2}$ the following expressions are obtained

$\dot{\mathrm{Q}}_{\mathrm{s} 1} P_{i l} / P_{\text {orb }}+\dot{\mathrm{Q}}_{\mathrm{s} 2} \bar{f}_{2}+\dot{\mathrm{Q}}_{p}+h_{i s}\left(\bar{T}_{i}-\bar{T}_{s}\right)-A_{s c} \varepsilon \sigma \overline{T_{s}^{4}}=0$

$Q_{i}=h_{i s}\left(\bar{T}_{i}-\bar{T}_{s}\right) P_{\text {orb }}$

where $\bar{f}_{2}=\frac{1}{P_{\text {orb }}} \int_{t}^{t+P_{\text {orb }}} f_{2}(t) \mathrm{d} t$ and $Q_{i}=\int_{t}^{t+P_{\text {orb }}} \dot{Q}_{i}(t) \mathrm{d} t$ is the net energy dissipated in the inner node in one period. Therefore, from Eqs. (7a) and $(7 \mathrm{~b})$ the temperature mean values are

$\bar{T}_{S}=\left(\frac{\dot{Q}_{T}}{A_{s c} \varepsilon \sigma}\right)^{1 / 4}$

$\bar{T}_{i}=\bar{T}_{s}+Q_{i} /\left(h_{i s} P_{o r b}\right)$

where $\dot{Q}_{T}=\dot{Q}_{s 1} P_{i l} / P_{\text {orb }}+\dot{Q}_{s} \bar{f}_{2}+\dot{Q}_{p}+Q_{i} / P_{\text {orb }}$. 
These expressions can be used either to make a preliminary assessment of the mean temperatures for a given satellite and orbit, or to design the appropriate configuration i.e. thermal coupling between nodes, possible use of heaters, estimation of energy needed to attain a given temperature level, etc. necessary to satisfy previously specified requirements on temperature limits.

\section{Fluctuations around the mean equilibrium temperatures}

From Eq. (4), making use of the mean temperature definitions (Eqs. (5) and (6)) and taking into account Eq. (7), the following equations are obtained for the temperature fluctuations

$$
\begin{aligned}
C_{s} \Delta \dot{T}_{s}=\dot{Q}_{s 1} & {\left[f_{1}(t)-\frac{P_{i l}}{P_{o r b}}\right]+\dot{Q}_{s 2}\left[f_{2}(t)-\bar{f}_{2}\right]+h_{i s}\left(\Delta T_{i}-\Delta T_{s}\right) } \\
& -4 A_{s c} \varepsilon \sigma \bar{T}_{s}^{3} \Delta T_{s} \\
\Delta T_{s}=\Delta T_{i} & +C_{i} \frac{\Delta \dot{T}_{i}}{h_{i s}}
\end{aligned}
$$

The problem can be stated for $\Delta T_{i}$ just by eliminating $\Delta T_{s}$ using Eqs. (9a) and (9b). To simplify the appearance of the resulting equation the following parameters are defined: $C=C_{s} / C_{i}$ is the ratio between the thermal capacities of the nodes; $h_{s s}=4 A_{s c} \varepsilon \sigma T_{s}^{3}$ represents the thermal coupling between the surface node and the outer space; $t_{i}=C_{i} / h_{i s}$ and $t_{s}=C_{s} / h_{s s}$ are the characteristic times of temperature variation due to the heat flux from node $i$ to node $s$ and from node $s$ to outer space, respectively; $\delta^{2}=t_{i} / t_{s}$ is the ratio of characteristic times, and $E=1+1 / C+\delta^{2}$ is the damping parameter. The terms representing the solar and albedo thermal loads can also be rewritten by defining $q_{1}(t)=\left[f_{1}(t)-P_{i l} / P_{o r b}\right]$ and $q_{2}(t)=$ $\beta\left[f_{2}(t)-\bar{f}_{2}\right]$, quantities that denote the deviation of the solar irradiation and albedo heat fluxes with respect to the average value along the orbit, where $\beta=\dot{Q}_{s 2} / \dot{Q}_{s 1}$. The substitution of these magnitudes in Eqs. (9a) and (9b) leads to

$\frac{C_{i}}{h_{i s}} \Delta \ddot{T}_{i}+E \Delta \dot{T}_{i}+\frac{h_{s s}}{C_{s}} \Delta T_{i}=\frac{\dot{Q}_{s 1}}{C_{s}}\left[q_{1}(t)+q_{2}(t)\right]$

The appearance of the equation can be further simplified by introducing the dimensionless temperatures $\theta_{s}, \theta_{i}$ and the dimensionless time $\tau$, defined as $\Delta T_{s}=\theta_{s} \Delta T_{c}, \Delta T_{i}=\theta_{i} \Delta T_{c}, t=\tau t_{c}$, where $\Delta T_{c}=\dot{Q}_{s 1} / h_{s s}$ is the characteristic temperature excursion from the mean value and $t_{c}=\left(t_{s} \cdot t_{i}\right)^{1 / 2}$ is the characteristic time of the combined problem. Furthermore, without solving the problem, $\Delta T_{c}$ gives the order of magnitude of the temperature fluctuations, which is of great interest in the conceptual and preliminary design phases. Eq. (10) can be rewritten in dimensionless form as follows

$\theta_{i}^{\prime \prime}+2 \zeta \theta_{i}^{\prime}+\theta_{i}=q_{1}(\tau)+q_{2}(\tau)$

where $\theta_{i}^{\prime}=\mathrm{d} \theta_{i} / \mathrm{d} \tau$ and $\zeta=E /(2 \delta)$ is the damping factor.

The roots of the characteristic equation of Eq. (11) are $r_{i, 12}=-\zeta \pm \sqrt{\zeta^{2}-1}$. From the definition of $\zeta$, it is deduced that $\zeta>0$, and therefore the system is stable since the equation does not have positive exponential solutions.

Given the external thermal loads $q_{1}(\tau), q_{2}(\tau)$ and suitable initial conditions, Eq. (11) can be solved to obtain $\theta_{i}(\tau)$. The outer nondimensional temperature can be obtained from Eq. (9b) as a function of the inner node temperature

$\theta_{s}=\theta_{i}+\delta \theta_{i}^{\prime}$

In this equation it can be seen that the ratio of characteristic times $\delta=\sqrt{\frac{C_{i}}{C_{s}} \frac{h_{s s}}{h_{i s}}}$ is a measure of the relative role of the thermal coupling between the internal and the external node. If $\delta<<1$, then $\theta_{s} \simeq \theta_{i}$ (strong coupling). If $\delta>>1$, then the coupling will be weak and there will be a greater difference between both temperatures. Therefore, $\delta$ is an outstanding parameter in the thermal design problem.

\section{Response to harmonic excitation}

\subsection{Governing equation and solution}

Eq. (11) can be rewritten as follows

$\theta_{i}^{\prime \prime}+2 \zeta \theta_{i}^{\prime}+\theta_{i}=q(\tau)$

with

$q(\tau)=q_{1}(\tau)+q_{2}(\tau)$

This simple equation is a classical one frequently found, among others, in the field of mechanical problems, as in the damped linear oscillator, which appears for instance in the forced vibration of damped systems or filter theory. Its terms have a clear physical meaning: $\theta_{i}^{\prime \prime}$ represents the effect of thermal inertia to temperature fluctuations, $2 \zeta \theta_{i}^{\prime}$ represents the damping of the system, $\theta_{i}$ the temperature restitution and $q(\tau)$ the generalized heat input driving the system. It should be remarked that Eq. (13) is obtained from a set of two first order ODEs, and the results should be traced back to it.

The thermal model can be reduced to the classical mass-springdamper system with the analogy between variables shown in Fig. 5. An interesting case is the response to harmonic excitation. The generalized heat input can be expressed as a Fourier series expansion, moreover, taking into account that the study has been focused on a periodic problem. According to the typical shape of each term contributing to the generalized heat input, shown previously in Fig. 2, the terms of Eq. (14) can been expressed as

$q_{1}(\tau)=\sum_{n=1}^{\infty} \frac{2}{\pi n} \sin (\pi \mu n) \cos \left(n \omega_{0} \tau\right)$
$\left.q_{2}(\tau)=\beta\left[\frac{1}{2} \cos \left(\omega_{0} \tau\right)-\sum_{n=1}^{\infty} \frac{2}{\pi\left(4 n^{2}-1\right)} \cos \left(2 n \omega_{0} \tau+n \pi\right)\right)\right]$

with $\mu=P_{i l} / P_{\text {orb }}$ and $\omega_{o}=\frac{2 \pi t_{c}}{P_{\text {orb }}}$.

The terms of these series tends to zero as $n$ increases. Thus, to help in a first approach, the first terms of each series will be the only ones retained. Therefore, Eq. (13) can be rewritten as

$\theta_{i}^{\prime \prime}+2 \zeta \theta_{i}^{\prime}+\theta_{i}=q_{o} \cos \left(\omega_{o} \tau\right)$

where $q_{0}=\left(\frac{2}{\pi} \sin (\pi \mu)+\frac{\beta}{2}\right)$.

The response to this excitation, once the transient period is over, will also be harmonic. The steady state response (particular solution of the differential equation) is not dependent on the initial conditions and therefore the validity of this study is universal. The steady state solution $\theta_{i P}$ of Eq. (17) can be written as

$\theta_{i P}(\tau)=\frac{q_{o}}{\sqrt{\left(1-\omega_{0}^{2}\right)^{2}+\left(2 \zeta \omega_{0}\right)^{2}}} \cos \left(\omega_{0} \tau-\varphi\right)$

where $\varphi=\tan ^{-1}\left(\frac{2 \zeta \omega_{0}}{1-\omega_{0}^{2}}\right)$.

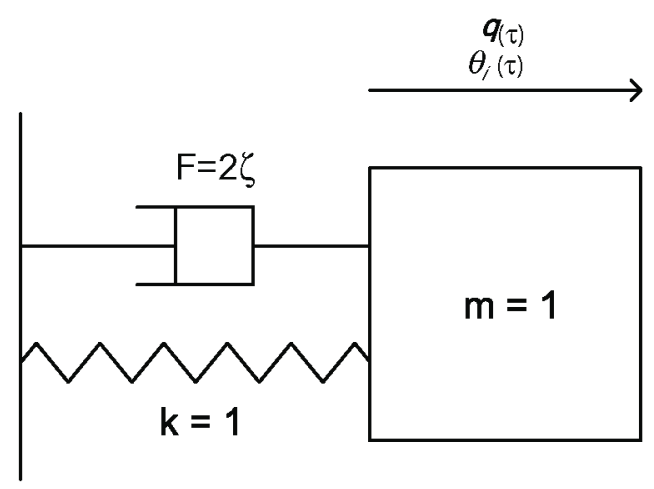

Fig. 5. Mechanical single degree of freedom system analogous to the thermal problem here analysed. 


\subsection{Analysis of the transfer function}

Working on the complex domain by applying the Fourier transform to Eq. (17), the magnitude of the transfer function that relates the inner node's temperature fluctuations to the heat input can be written as follows:

$\left|F_{\theta_{i} q}\right|=\left|\frac{\Theta_{i}}{Q}\right|=\left[\left(1-\omega_{o}^{2}\right)^{2}+4 \zeta^{2} \omega_{o}^{2}\right]^{-1 / 2}$

and the phase delay between input and output is

$\varphi_{\theta_{i} q}=\tan ^{-1}\left(\frac{2 \zeta \omega_{o}}{1-\omega_{o}^{2}}\right)$

The characteristics of the transfer function can be better appraised by studying the damping term. From the definition of $\zeta$ :

$\zeta^{2}=\frac{1}{4} \frac{E^{2}}{\delta^{2}}=\frac{1}{4} \frac{(1+\mathrm{C}+H)^{2}}{C H}$

where $H=h_{S S} / h_{i s}$ is the ratio of outer node-space thermal coupling to inner node-outer node thermal coupling, as defined above. From this definition the parameter $\delta$ can be rewritten as follows:

$\delta=\sqrt{\frac{H}{C}}$

From Eq. (20) it can be seen that the function $\zeta^{2}(H, C)$ is symmetric on $H$ and $C$. Thus, it is sufficient to study its dependence on one of the parameters. The variation of $\zeta^{2}$ with $H$ for different values of $C$ is shown in Fig. 6. For a given value of $C$, the minimum value of $\zeta^{2}$ is

$$
\zeta_{m}^{2}=\zeta^{2}\left(H_{m}, C\right)=1+\frac{1}{C}
$$

which occurs when $H=H_{m}=1+C$. The absolute minimum value of $\zeta=\zeta_{m m}=1$ takes place for $C \rightarrow \infty$ and, therefore, the condition $\zeta^{2}>1 / 2$ is fulfilled for every value of $H$ and $C$. Accordingly, the case under study corresponds to an overdamped system, that is, no resonant behaviour in $\theta_{i}$ can be expected as in the case of underdamped systems.

Note also in Fig. 6 that the same damping factor $\zeta$ can be reached with the same ratio of thermal inertia $C$ for two very different values of the thermal coupling ratio $H$.

In this situation the module of the transfer function $F_{\theta_{i} q}$ (Eq. (19a)) can be plotted as a function of $\omega_{o}$ for different values of $\zeta$,

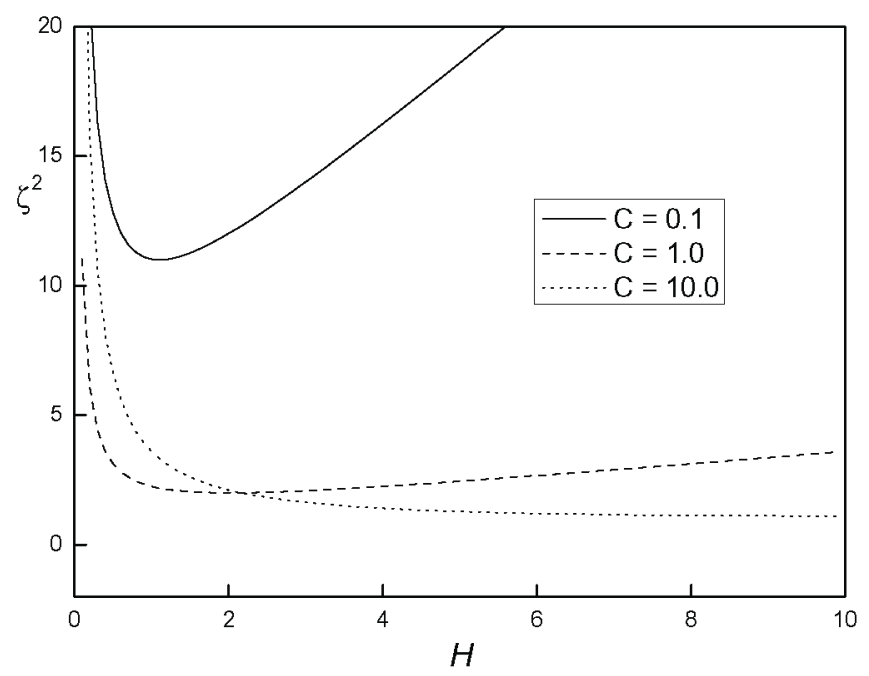

Fig. 6. Damping factor as a function of the ratio of thermal couplings $H$ for different values of ratio of thermal capacities $C$. as shown in Fig. 7. There is no amplitude resonance as $\zeta>1 / \sqrt{2}$. Observe the decay of the oscillations amplitude as $\omega_{0}$ increases.

Regarding the phase delay between the external heat input and the inner node's temperature fluctuations $\varphi_{\theta_{i} q}$, as is shown in Fig. 8, it tends to zero for small values of $\omega_{o}$. Recalling the definition $\omega_{o}=\frac{2 \pi t_{c}}{P_{\text {orb }}}=\frac{2 \pi}{P_{\text {orb }}} \sqrt{\frac{C_{i} C_{s}}{h_{i s} h_{s s}}}$, these low values can be obtained:

(a) For long-period orbits, i.e. high altitude orbits.

(b) For satellites with low thermal capacities.

(c) For satellites with strong thermal couplings between the inner and the outer components and with the exterior.

A phase difference of $\pi$ radians between input and output takes place for the opposite situation, that is:

(a) For short-period orbits, i.e. low altitude orbits.

(b) For satellites with high thermal capacities.

(c) For satellites with weak thermal couplings between the inner and the outer components and with the exterior.

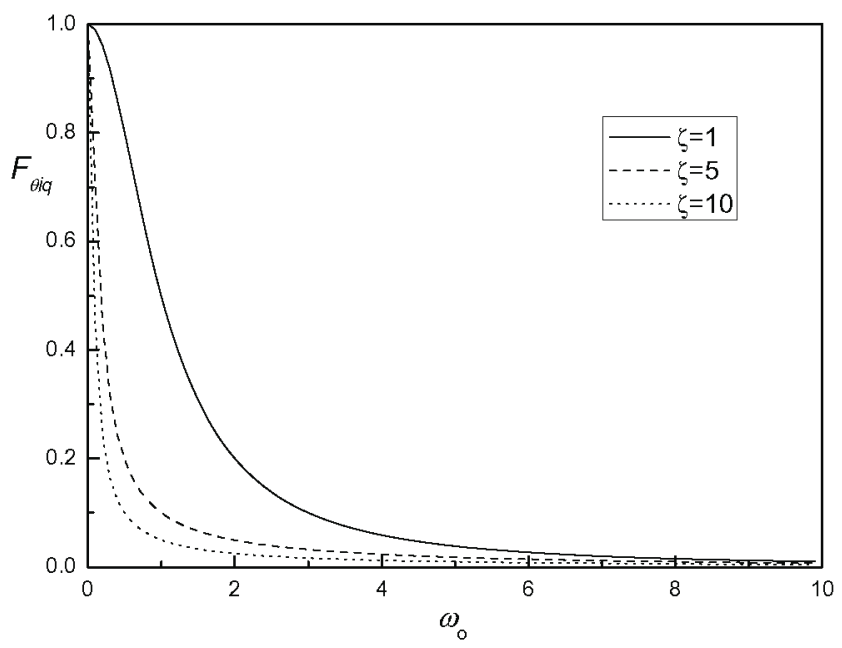

Fig. 7. Transfer function that relates the inner node's temperature fluctuations with the heat input as a function of the frecuency $\omega_{0}$ for different values of $\zeta$.

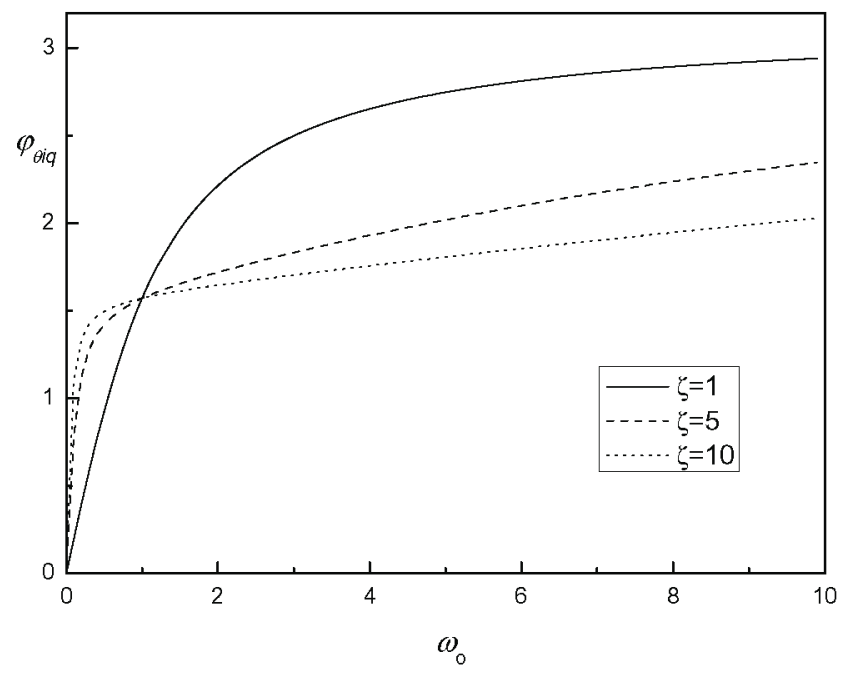

Fig. 8. Phase delay between the external heat input and the inner node's temperature fluctuations. 
A phase quadrature takes place for $\omega_{o}=1$, where in this case $\varphi=\pi / 2$.

Therefore, after the analysis of the response of the system to a given excitation, for a given orbit the satellite parameters $C_{i}, C_{s}$, $h_{i s}, h_{s s}$ can be chosen to reproduce the desired behaviour.

\subsection{Thermal coupling between nodes}

Starting from Eq. (12), and following a method analogous to the one used in the previous subsections, a second transfer function $J$ can be obtained to relate the temperature oscillation amplitude of the inner node to that of the outer node. This function is:

$\left|U_{\theta_{s} \theta_{i}}\right|=\left|\frac{\Theta_{S}}{\Theta_{i}}\right|=\left[1+\left(\delta \omega_{0}\right)^{2}\right]^{1 / 2}$

and the phase delay is $\varphi_{\theta_{s} \theta_{i}}=\tan ^{-1}\left(\delta \omega_{o}\right)$.

Fig. 9 shows the magnitude of this transfer function and Fig. 10 shows the phase delay between the temperature fluctuation of both nodes. For $\delta \rightarrow \infty$ the coupling between nodes is weak, the amplitude of the temperature oscillations is much higher in the shell than in the inner node, $\theta_{s}>>\theta_{i}$, and the phase

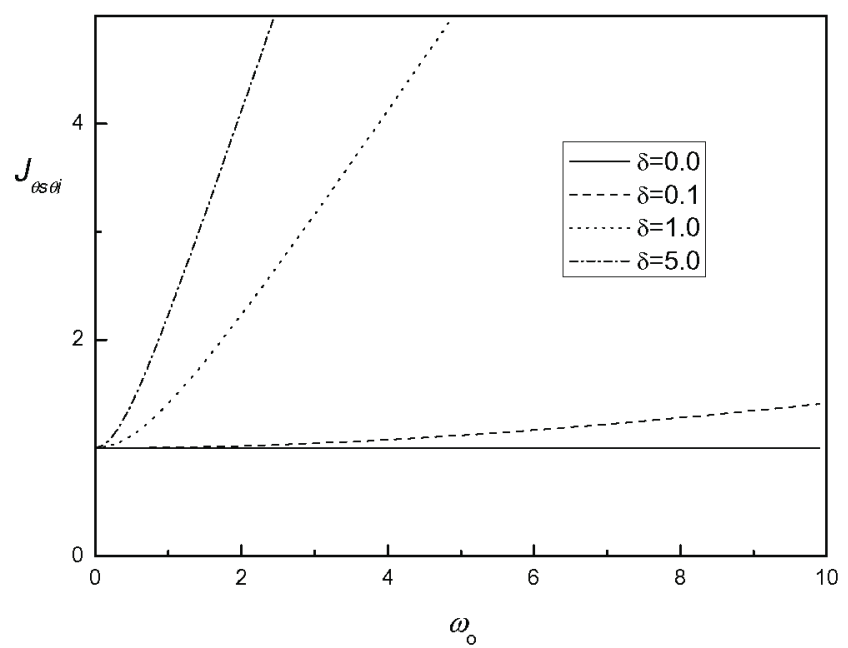

Fig. 9. Transfer function that relates both nodes' temperature fluctuations for different values of $\delta$.



Fig. 10. Phase delay between both nodes' temperature fluctuations.

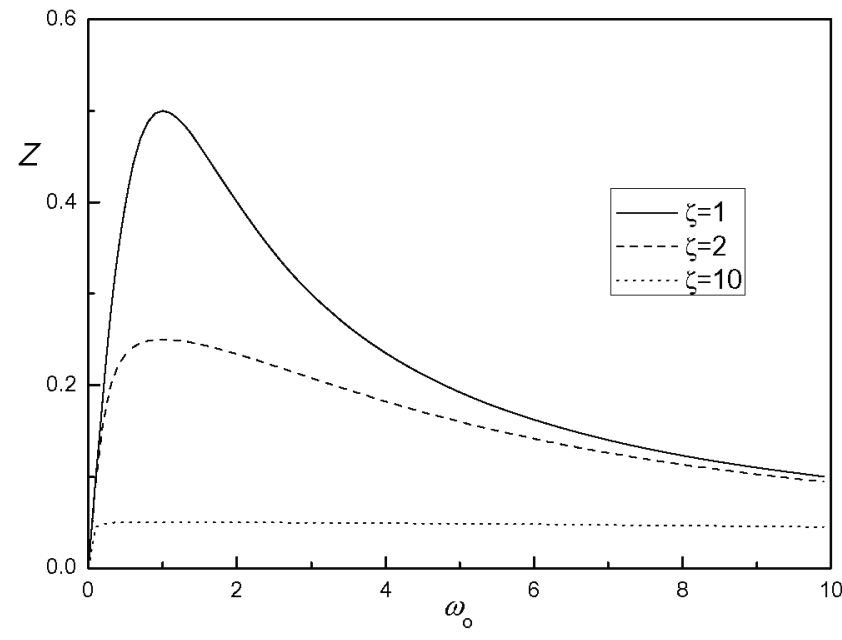

Fig. 11. Transfer function that relates the thermal gradients with the heat input as a function of the frecuency $\omega_{0}$ for different values of $\zeta$.

delay is $\pi / 2$. For small values of $\delta$, that is for $\delta \rightarrow 0$, there is a strong coupling between nodes, $\theta_{s} \cong \theta_{i}$, both in amplitude and phase. In this case the outer node's temperature fluctuations instantaneously respond to those of the inner node and vice versa.

Recalling the definition $\delta=(H / C)^{1 / 2}$, it can be concluded again that the parameters contributing to its value can be appropriately selected to obtain the desired result.

\subsection{Thermal gradients}

In the previous subsection the transfer function that relates the temperature fluctuations of the two nodes under study has been obtained. Another interesting result is obtained by calculating the ratio between the difference of temperature fluctuations of both nodes and the heat input into the system: $\left(\Theta_{s}-\Theta_{i}\right) / Q$. This is a measure of how the oscillations of the heat loads onto the satellite are translated into oscillations of temperature gradients within the system. This is also of great interest in the design process as in many cases the thermal gradients will be constrained within certain allowable limits, derived from system requirements (e.g. optical quality of images). Taking into account the definition of the previously obtained transfer functions $F_{\theta_{i} q}$ and $J_{\theta_{s} \theta_{i}}$, the searched term can be expressed as: $\left(\Theta_{s}-\Theta_{i}\right)$ ) $Q=F_{\theta_{i} q}\left(U_{\theta_{s} \theta_{i}}-1\right)$. Thus, a new transfer function $Z$ can be defined. Its magnitude is:

$Z=\left|\left(\Theta_{s}-\Theta_{i}\right) / Q\right|=\delta \omega_{o}\left[\left(1-\omega_{o}^{2}\right)^{2}+4 \zeta^{2} \omega_{o}^{2}\right]^{-1 / 2}$

This function is plotted in Fig. 11 as a function of $\omega_{o}$ for different values of the damping factor $\zeta$ and a value of $\delta=1$. It can be shown that a resonance appears as $Z$ reaches a maximum $Z_{\max }$ for values of dimensionless frequency $\omega_{0}=1$ (it does not depend on $\zeta$ ), being $Z_{\max }=\delta /(2 \zeta)$. As $\zeta_{\min }=1$, the maximum absolute amplification is $Z_{\max }=\delta / 2$. This means that the amplitude of the thermal gradient oscillations are maximum at this frequency and, therefore, it will have to be avoided if there is a limit in the allowed gradients. Returning again to the definition $\omega_{0}=\frac{2 \pi}{P_{\text {orb }}} \sqrt{\frac{C_{2} C_{s}}{h_{\text {is }} h_{\text {ss }}}}$, the heat capacities and the thermal couplings have to be appropriately selected according to the orbital period to avoid this amplification of the gradients. Note also in Fig. 11 that this effect decreases as the damping factor increases. The influence of the parameter $\delta$ is simply a scale factor in Fig. 11. 


\section{Thermal stability to random perturbations}

A possible analytical approach to verify the thermal stability requirements of a satellite's system consists in studying the response of the system to random fluctuations of the thermal loads. This study is carried out by means of a series of transfer functions that relate the temperature fluctuations of the satellite's nodes to a randomly fluctuating environment.

A statistical characterization of the response to this kind of input can be obtained by means of the power spectral density (PSD). For the inner node's temperature the PSD is defined as $\operatorname{PSD}_{\theta_{i}}=\Theta_{i}(\omega) \cdot \Theta_{i}(\omega)^{*}=\left|\Theta_{i}(\omega)\right|^{2}$, where the symbol * stands for the complex conjugate. This product appears when trying to obtain the mean square of the response, given by:

$\psi_{\theta_{i}}^{2}=\frac{1}{P_{\text {orb }}} \int_{P_{\text {orb }}} \theta_{i}(t) \cdot \theta_{i}(t) \mathrm{d} t$

Substituting in Eq. (24) the inverse Fourier transform, and applying the Parseval's theorem yields

$\psi_{\theta_{i}}^{2}=\frac{1}{2 \pi} \int_{-\infty}^{\infty} \operatorname{PSD}_{\theta_{i}} \mathrm{~d} \omega$

Usually there will be a frequency band of interest, so that

$\psi_{\theta_{i}}^{2}\left(\omega_{1}, \omega_{2}\right)=\frac{1}{2 \pi} \int_{\omega_{1}}^{\omega_{2}} \operatorname{PSD}_{\theta_{i}} \mathrm{~d} \omega$

The term $\psi_{\theta_{i}}^{2}$ is also the variance of the temperature fluctuations since its mean value is zero. Therefore, the area under the curve $\operatorname{PSD}(\omega)$ will give the square of the standard deviation. This formulation is needed to analyse some thermal problems. For instance, as mentioned above, some scientific payloads have thermal specifications defined in terms of $K / \sqrt{\mathrm{Hz}}$ in a given frequency range, sometimes derived from dimensional stability.

Since the PSDs of the nodes' temperatures fluctuations are not known, the method to obtain them consists of establishing their relation with the PSD of the thermal loads through the transfer functions obtained in the previous sections. The substitution of $\Theta_{i}=F_{\theta_{i}} Q$ in Eq. (26) results

$\operatorname{PSD}_{\theta_{i}}(\omega)=\left|F_{\theta_{i} q}(\omega)\right|^{2} \operatorname{PSD}_{q}(\omega)$

and following a similar path

$\operatorname{PSD}_{\theta_{s}}(\omega)=\left.V_{\theta_{s} \theta_{i}}(\omega)\right|^{2} \operatorname{PSD}_{\theta_{i}}(\omega)$

From these last expressions the outer node's temperature can be related to the heat input as

$\operatorname{PSD}_{\theta_{s}}(\omega)=\left.\left|F_{\theta_{i} q}(\omega)\right|^{2} U_{\theta_{s} \theta_{i}}(\omega)\right|^{2} \operatorname{PSD}_{q}(\omega)$
Therefore, from the environment perturbations, if the transfer functions are known, the PSD of the temperature fluctuations can be directly calculated and, accordingly, the stability requirements can be verified.

\section{Conclusions}

The problem of the thermal behaviour of a small satellite has been analysed by using a two-node model. In order to obtain general results for different types of satellites and orbits, the equations have been reduced to a second order non-homogeneous differential equation for the inner node's temperature fluctuations around its mean value. Some dimensionless parameters have been identified, which have allowed us to obtain valuable conclusions from the analysis of the response to a harmonic excitation. It has been shown that the system is overdamped. The damping factor has been determined as a combination of the satellite thermal properties (heat capacities and thermal couplings). For a given orbit, several transfer functions have been analysed: the internal temperature fluctuations response to external thermal loads, the relation between the inner and outer temperature fluctuations, and the thermal gradient fluctuation response to external heat loads. In the last case, a resonant behaviour has been found. It has been shown that it is possible to appropriately select the heat capacities and thermal couplings of the satellite in order to obtain the desired result.

Those straightforward equations are also useful to streamline the verification of thermal stability requirements of small satellites in the frequency domain.

\section{Acknowledgements}

The authors are grateful to the Thermal Control Division of EADS-CASA-Espacio for their support and fruitful discussions. This work has been supported by the Spanish Ministerio de Educación y Ciencia, Project ESP2006-13030-C06-05.

\section{References}

[1] D.G. Gilmore, Spacecraft Thermal Control Handbook, The Aerospace Corporation, 2002

[2] K. Oshima, Y. Oshima, An analytical approach to the thermal design of spacecraft, Institute of Space and Aeronautical Science of Tokyo, Report No. 419, 1968.

[3] C. Arduini, G. Laneve, S. Folco, Linearized techniques for solving the inverse problem in the satellite thermal control, Acta Astronautica 43 (1998) 473-479.

[4] M.A. Gadalla, Prediction of temperature variation in a rotating spacecraft in space environment, Applied Thermal Engineering 25 (2005) 2379-2397.

[5] A.A. Shabana, Theory of Vibration: An Introduction, Springer, New York, 1995.

[6] G. Barbagallo, D. Stramaccioni, Use of Spectral Analysis in Thermal Stability Verification, SAE Technical Paper No. 2002-01-2373, 2002.

[7] V.L. Pisacane, R.C. Moore, Fundamentals of Space Systems, Oxford University Press, 1994.

[8] P. Fortescue, J. Stark, G. Swinerd, Spacecraft Systems Engineering, 3rd ed., John Wiley \& Sons, 2003. 